\title{
Limitations of resting haemodynamics in chronic thromboembolic disease without pulmonary hypertension
}

\author{
To the Editor:
}

There is renewed interest in the haemodynamic definitions of pulmonary hypertension $(\mathrm{PH})$, reigniting an old debate about diagnostic thresholds [1]. Recent prospective data support work dating back over 40 years demonstrating patients with "borderline" $\mathrm{PH}$ (mean pulmonary artery pressure (mPAP) $<25 \mathrm{mmHg}$ ) can still have significant morbidity and mortality [2]. Therefore, lowering the mPAP threshold for the diagnosis of precapillary pulmonary arterial hypertension (PAH) has been discussed at World Symposium on Pulmonary Hypertension in Nice, France, in 2018. A potentially different approach has arisen in group 4 (chronic thromboembolic pulmonary hypertension (CTEPH)), where the concept of chronic thromboembolic disease without PH (CTED) has gained traction. This describes a population of patients with mPAP $<25 \mathrm{mmHg}$, with no lower limit, who have persistent vascular obstructions, impaired response to exercise, and a high impact of disease on symptoms and quality of life (QoL). The 25-mmHg threshold is important partly because it excludes patients who might benefit from treatment, and then precludes their participation in clinical trials, forming a cycle that prevents regulatory approved treatment in the future. In the CTED to CTEPH spectrum, it is unclear if reducing the threshold is the best way to address this inequity, as minimal data exists detailing outcomes $<25 \mathrm{mmHg}$. In the UK, we have undertaken pulmonary endarterectomy (PEA) on a selected, symptomatic cohort of operable CTED patients with good results [3], which were recapitulated by others [4, 5]. A valid criticism of our previous work [3] is the retrospective, selective nature of the subjects and a lack of understanding about the natural history of the disease without treatment. Here, we present the first prospective cohort of patients with operable CTED (institutional review board project reference S02297), and hypothesised that clinically meaningful symptoms, limitation and physiology would relate to haemodynamics. Royal Papworth Hospital (Cambridge, UK) is the national PEA referral centre and to minimise tertiary speciality referral bias, we have included only regional nonspecialist referrals. Regional incident cases referred in 2015-2017 with suspected CTED/CTEPH were prospectively assessed. All patients were reviewed at the national CTEPH multidisciplinary team (MDT) meeting. Patients with operable CTED underwent standard CTEPH investigations [6] with additional exercise right heart catheterisation (RHC) and incremental cardiopulmonary exercise testing (CPET) [7]. The zero reference was set at the midthoracic level. During exercise RHC, patients were asked to pedal for $5 \mathrm{~min}$ at $40 \%$ of the workload achieved during incremental CPET (load range 9-104 W, maximal supine exercise test could not been performed due to technical limitation of ergometer). The mPAP, pulmonary wedge pressure (measured over three breath cycles, when feasible), mixed venous saturation, heart rate and systemic blood pressure were measured, followed by cardiac output (CO) measurement using the thermodilution technique. Pulmonary vascular resistance, total pulmonary resistance, cardiac index, pulmonary artery compliance and mPAP-CO slope were calculated. All patients diagnosed with operable CTED were followed up for a minimum of 1 year.

Baseline characteristics were expressed as numbers and percentages for categorical variables and mean $\pm \mathrm{SD}$ or median (interquartile range) for continuous variables according to data distribution. Between-group and within-group comparisons were made using the parametric and nonparametric test as appropriate. A p-value of $<0.05$ was considered significant. Correction for multiple testing where necessary was performed, and both adjusted and unadjusted p-values were reported. Statistical analysis was performed with R (www.r-project.org).

@ERSpublications

Chronic thromboembolic disease (CTED) without pulmonary hypertension is emerging as a new and important disease classification http://ow.ly/ZmK730mv5Xh

Cite this article as: Swietlik EM, Ruggiero A, Fletcher AJ, et al. Limitations of resting haemodynamics in chronic thromboembolic disease without pulmonary hypertension. Eur Respir J 2019; 53: 1801787 [https:// doi.org/10.1183/13993003.01787-2018]. 
Out of 176 patients referred for suspected CTED/CTEPH, 34 were diagnosed with CTED and 125 with CTEPH. An alternative diagnosis was made in the remaining 17 (multifactorial pulmonary hypertension, $\mathrm{n}=7$; asymptomatic with proximal persistent perfusion defects, $\mathrm{n}=6$; asymptomatic with distal persistent perfusion defects, $\mathrm{n}=2$; heart failure with preserved ejection fraction, $\mathrm{n}=1$; severe aortic stenosis, $\mathrm{n}=1$ ). CTED patients had technically operable disease. All patients were treated for $\geqslant 3$ months with anticoagulation and were PAH-targeted therapy naïve. CTED patients were younger than those with CTEPH (54 (39-66) versus 66 (55-73) years, $\mathrm{p}=0.001$ ), had a better functional status (World Health Organization (WHO) class I/II 50\% versus $19 \%, \mathrm{p}<0.001$ ) and higher 6 -min walk test $(6 \mathrm{MWT})$ distance ( $396 \pm 123$ versus $278 \pm 119 \mathrm{~m}, \mathrm{p}<0.001)$. In CTED, total pulmonary vascular obstruction index (TPVOI) [8] was lower $(33 \pm 14 \%$ versus $43 \pm 14 \%, \mathrm{p}<0.001)$ and modestly correlated with $\mathrm{mPAP}(\mathrm{r}=0.25, \mathrm{p}<0.001)$.

15 patients had a resting mPAP between 21 and $24 \mathrm{mmHg}$, and 19 had $\mathrm{mPAP} \leqslant 20 \mathrm{mmHg}$. There were no between-group differences in age, body mass index, comorbidities, WHO class, 6MWT distance, symptom scoring or functional assessment. Exercise RHC at $40 \%$ of maximal load $(n=25)$ demonstrated an increase from baseline in mPAP and CO, and drop of total pulmonary resistance (table 1). There was a strong correlation between resting and exercise mPAP $(r=0.66(0.36-0.84), p=0.004)$. Six of the 34 patients were offered and accepted surgical treatment. The decision regarding surgery was made in the CTEPH MDT meeting, and was independent of the study and based on the results of clinical tests, QoL, comorbidities, risk-benefit ratio and patient preferences. Operated patients were characterised by lower peak oxygen consumption $\left(V^{\prime} \mathrm{O}_{2}\right)$ and peak oxygen pulse, higher ventilatory equivalents for carbon dioxide $\left(V^{\prime} \mathrm{E} / V^{\prime} \mathrm{CO}_{2}\right)$ at anaerobic threshold (AT) and TPVOI, and worse self-reported QoL (table 1). There was no operative mortality in this cohort. Nonoperated patients were followed up for a minimum of 1 year. None of the patients were treated with PAH-targeted therapy but all were anticoagulated. In nonoperated patients, Cambridge Pulmonary Hypertension Outcome Review (CAMPHOR) symptoms (11 (5-16) versus 1 year 9 (6-15)) and QoL (5 (1-12) versus 6 (4-11)) did not change. There were no significant differences in peak

TABLE 1 Cohort characteristics

\begin{tabular}{|c|c|c|c|c|c|c|c|}
\hline & All & \multicolumn{3}{|c|}{ mPAP } & \multicolumn{3}{|c|}{ Surgery } \\
\hline Patients & 34 & 15 & 19 & & 28 & 6 & \\
\hline Age years & $53 \pm 17$ & $58 \pm 16$ & $48 \pm 17$ & 0.087 & $55 \pm 17$ & $43 \pm 14$ & 0.103 \\
\hline WHO FC & & & & 0.187 & & & 0.842 \\
\hline 1 & $4(12 \%)$ & $0(0 \%)$ & $4(21 \%)$ & & $4(14 \%)$ & $0(0 \%)$ & \\
\hline 2 & $13(38 \%)$ & $7(47 \%)$ & $6(32 \%)$ & & $10(36 \%)$ & $3(50 \%)$ & \\
\hline Symptom & $11 \pm 7$ & $12 \pm 5$ & $10 \pm 8$ & 0.308 & $10 \pm 7$ & $14 \pm 5$ & 0.141 \\
\hline Activity & $6(3-12)$ & $7(5-13)$ & $3(2-11)$ & 0.139 & $6(2-11)$ & $6(4-11)$ & 0.833 \\
\hline QoL & $5(1-13)$ & $6(3-13)$ & $5(0-14)$ & 0.556 & $4(0-12)$ & $14(9-16)$ & 0.030 \\
\hline $\mathrm{BMI} \mathrm{kg} \cdot \mathrm{m}^{-2}$ & $29 \pm 5$ & $30 \pm 4$ & $28 \pm 6$ & 0.307 & $29 \pm 6$ & $28 \pm 3$ & 0.643 \\
\hline 6MWT distance $\mathrm{m}$ & $402 \pm 114$ & $389 \pm 115$ & $412 \pm 116$ & 0.598 & $401 \pm 116$ & $404 \pm 118$ & 0.955 \\
\hline Peak $V^{\prime} \mathrm{O}_{2} \%$ predicted & $88 \pm 17$ & $83 \pm 11$ & $91 \pm 20$ & 0.142 & $92 \pm 16$ & $70 \pm 10$ & $0.001^{\#}$ \\
\hline PVR WU & $1.9(1.4-2.4)$ & $2.3(2.0-2.8)$ & $1.4(1.2-1.8)$ & $0.003^{\#}$ & $1.7(1.3-2.2)$ & $2.5(2.2-2.6)$ & 0.061 \\
\hline TPR WU & $3.7(3.1-4.2)$ & $4.1(3.7-4.8)$ & $3.2(2.8-3.7)$ & $0.001^{\#}$ & $3.6(3.0-4.0)$ & $4.2(4.0-4.4)$ & 0.071 \\
\hline $\mathrm{PAC} \mathrm{mL} \cdot \mathrm{mmHg}^{-1}$ & $4.1(2.8-5.6)$ & $3(2.6-4.4)$ & $4.7(3.9-5.7)$ & 0.058 & $4.4(2.8-5.6)$ & $3.8(2.9-4.0)$ & 0.633 \\
\hline Exercise mPAP $\mathrm{mmHg}$ & $29 \pm 7$ & $34 \pm 7$ & $26 \pm 5$ & $0.006^{\#}$ & $30 \pm 8$ & $28 \pm 3$ & 0.582 \\
\hline Exercise TPR WU & $2.7(2.2-3.6)$ & $3.5(2.6-3.7)$ & $2.3(2.1-2.9)$ & 0.022 & $2.9(2.2-3.7)$ & $2.6(2.3-2.9)$ & 0.505 \\
\hline Exercise $\mathrm{CO} L \cdot \mathrm{min}^{-1}$ & $10.4 \pm 3.1$ & $10.0 \pm 2.1$ & $10.9 \pm 3.9$ & 0.497 & $10.4 \pm 3.4$ & $10.9 \pm 1.4$ & 0.591 \\
\hline mPAP-CO slope & $1.6(1.1-3.4)$ & $2.8(1.4-3.7)$ & $1.5(1.1-2.5)$ & 0.301 & $2.5(1.4-4.0)$ & $1.1(1.0-1.2)$ & 0.088 \\
\hline TPVOI \% & $34 \pm 14$ & $37 \pm 13$ & $31 \pm 15$ & 0.287 & $31 \pm 13$ & $51 \pm 6$ & $0.001^{\#}$ \\
\hline
\end{tabular}

Data are presented as mean $\pm S D$ or median (interquartile range), unless otherwise stated. mPAP: mean pulmonary artery pressure; WHO: World Health Organization; FC: functional class; CAMPHOR: Cambridge Pulmonary Hypertension Outcome Review; QoL: quality of life; BMI: body mass index; 6MWT: 6-min walk test; $V^{\prime} \mathrm{O}_{2}$ : oxygen consumption; $V^{\prime} \mathrm{E} / \mathrm{V}^{\prime} \mathrm{CO}_{2}$ : ventilatory equivalent for carbon dioxide; AT: anaerobic threshold; $\mathrm{CO}$ : cardiac output; PAC: pulmonary artery compliance; PVR: pulmonary vascular resistance; WU: Wood units; TPR: total pulmonary resistance; TVPOI: total pulmonary vascular obstruction index. ${ }^{\#}$ : significant after false discovery rate correction. 
$V^{\prime} \mathrm{O}_{2}(94 \%(83-100 \%)$ versus 1 year $104 \%$ (83-110\%) predicted), 6MWT distance (388 (362-460) versus $419(330.5-511) \mathrm{m})$ or $\mathrm{N}$-terminal pro-brain natriuretic peptide $\left(55(32-82)\right.$ versus $\left.65(36-112) \mathrm{pg} \cdot \mathrm{dL}^{-1}\right)$. Two patients died within 1 year, both malignancy related. One patient delayed follow-up for treatment of a new cancer and six patients were clinically stable, electing to have follow-up at their local centre. At 3-6 months follow-up, operated patients showed haemodynamic (mPAP $21 \pm 2$ versus $16 \pm 3 \mathrm{mmHg}, \mathrm{p}=0.007$ ), symptomatic (CAMPHOR symptoms $14 \pm 5$ versus $7 \pm 6, \mathrm{p}=0.029$ ) and functional (6MWT distance $404 \pm 118$ versus $454 \pm 109 \mathrm{~m}, \mathrm{p}=0.006)$ improvement. Furthermore, CPET ventilatory measures also improved ( $V^{\prime} \mathrm{E} / V^{\prime} \mathrm{CO}_{2}$ at $\mathrm{AT} 42 \pm 5$ versus $33 \pm 5 \mathrm{mmHg}, \mathrm{p}=0.003$; end-tidal carbon dioxide tension at $\mathrm{AT} 27 \pm 3$ versus $34 \pm 3 \mathrm{mmHg}, \mathrm{p}=0.009$ ).

This is the first prospective cohort of patients systematically assessed for operable CTED with medium-term follow-up. Most patients are not offered surgery and remain symptomatic, but are clinically and objectively stable; therefore, treatment options can be carefully considered. Despite the small cohort of patients undergoing PEA, there were significant differences, consistent with our previous reports, in baseline physiology on exertion and CAMPHOR scores in QoL [3, 9-11].

We are therefore operating on a subgroup of patients where resting haemodynamics are of limited use, but who have worse pathological and physiological impairment on exercise, and who self-report a lower quality of life. Although resting haemodynamics are critical to establishing the diagnosis of $\mathrm{PH}$, a more lenient definition of $\mathrm{PH}$ to $20 \mathrm{mmHg}$ will be of debatable additional benefit as symptoms and abnormal physiology do not relate to resting pressures or pulmonary resistance. Consistent with our retrospective study where $48 \%$ of the operated population had an mPAP of $\leqslant 20 \mathrm{mmHg}$, in our prospective study of predominantly nonoperated patients, this was $56 \%$. With the advent of balloon pulmonary angioplasty, medical therapeutic options and physical rehabilitation programmes, there is now a need for consensus on disease classification. International prospective registry data will be critical in guiding this effort. Our data reinforce the concept that for technically operable CTED/CTEPH patients, lowering the haemodynamic thresholds may still miss symptomatic patients with abnormal physiology. Notably, all but one of our patients were under the $3 \mathrm{WU}$ threshold. We demonstrate that patients can have a clinically meaningful burden of disease with impaired physiology and report significant symptoms in the context of "normal" resting haemodynamics. Studies in this group of patients will need a different trial design from traditional PAH and CTEPH studies. Resting haemodynamics and 6MWT are unlikely to be feasible end-points, time to clinical worsening will not be useful, and more extensive exercise physiology measurements will be required to fully interrogate symptomatic limitation.

Emilia Maria Swietliki, ${ }^{1,2,3}$, Alessandro Ruggiero ${ }^{1}$, Andrew J. Fletcher ${ }^{1}$, Dolores Taboada ${ }^{1}$, Emily Knightbridge ${ }^{1}$, Louise Harlow ${ }^{1}$, Ian Harvey ${ }^{1}$, Nicholas Screaton ${ }^{1}$, John E. Cannon ${ }^{1}$, Karen K.K. Sheares ${ }^{1}$, Choo Ng $^{1}$, David P. Jenkins ${ }^{1}$, Joanna Pepke-Zaba ${ }^{1}$ and Mark R. Toshner ${ }^{1,2}$

${ }^{1}$ Royal Papworth Hospital NHS Foundation Trust, Cambridge, UK. ${ }^{2}$ University of Cambridge, Dept of Medicine, Cambridge, UK. ${ }^{3}$ University of Warmia and Mazury, Olsztyn, Poland.

Correspondence: Mark R. Toshner, Level 5, Dept of Medicine, University of Cambridge, Addenbrooke's Hospital, Cambridge, CB2 0QQ, UK. Email: mrt34@medschl.cam.ac.uk

Received: Aug 132018 | Accepted after revision: Oct 182018

Conflict of interest: E.M. Swietlik reports receiving an educational/travel grant from AOP ORPHAN outside the submitted work. A. Ruggiero has nothing to disclose. A.J. Fletcher has nothing to disclose. D. Taboada reports receiving speaker's honoraria and education/travel grants from Actelion, Bayer, GlaxoSmithKline, Lilly, MDS and Pfizer outside the submitted work. E. Knightbridge has nothing to disclose. L. Harlow has nothing to disclose. I. Harvey has nothing to disclose. N. Screaton has nothing to disclose. J.E. Cannon reports receiving educational grants to organise education meetings from Actelion, MSD and GSK, outside the submitted work. K.K.K. Sheares reports receiving educational support (travel, registration and accommodation) to attend conferences from Actelion, Bayer, MSD and GSK, and an honorarium for consulting from Actelion, outside the submitted work. C. Ng has nothing to disclose. D.P. Jenkins reports receiving honoraria for speaking on PTE surgery from Bayer/MSD and consultancy fees as adjudicator for the MERIT trial from Actelion, outside the submitted work. J. Pepke-Zaba or her institution have received research/ educational grants from Actelion, Merck, Bayer and GSK; and J. Pepke-Zaba has served on advisory boards for Actelion, Merck, Bayer and GSK. M.R. Toshner reports receiving speaker's honoraria and travel grants from Acetlion, Bayer, GlaxoSmithKline and MSD, outside of the submitted work.

\section{References}

1 Maron BA, Wertheim BM, Gladwin MT. Under pressure to clarify pulmonary hypertension clinical risk. Am J Respir Crit Care Med 2018; 197: 423-426.

2 Douschan P, Kovacs G, Avian A, et al. Mild elevation of pulmonary arterial pressure as a predictor of mortality. Am J Respir Crit Care Med 2018; 197: 509-516.

3 Taboada D, Pepke-Zaba J, Jenkins DP, et al. Outcome of pulmonary endarterectomy in symptomatic chronic thromboembolic disease. Eur Respir J 2014; 44: 1635-1645. 
4 van Kan C, van der Plas MN, Reesink HJ, et al. Hemodynamic and ventilatory responses during exercise in chronic thromboembolic disease. J Thorac Cardiovasc Surg 2016; 152: 763-771.

5 Guth S, Wiedenroth $\mathrm{CB}$, Rieth A, et al. Exercise right heart catheterisation before and after pulmonary endarterectomy in patients with chronic thromboembolic disease. Eur Respir J 2018; 52: 1800458.

6 Galie N, Humbert M, Vachiery JL, et al. 2015 ESC/ERS Guidelines for the diagnosis and treatment of pulmonary hypertension. Eur Respir J 2015; 46: 903-975.

7 American Thoracic Society, American College of Chest Physicians. ATS/ACCP Statement on cardiopulmonary exercise testing. Am J Respir Crit Care Med 2003; 167: 211-277.

8 Hoey ET, Mirsadraee S, Pepke-Zaba J, et al. Dual-energy CT angiography for assessment of regional pulmonary perfusion in patients with chronic thromboembolic pulmonary hypertension: initial experience. AJR Am J Roentgenol 2011; 196: 524-532.

9 McCabe C, Deboeck G, Harvey I, et al. Inefficient exercise gas exchange identifies pulmonary hypertension in chronic thromboembolic obstruction following pulmonary embolism. Thromb Res 2013; 132: 659-665.

10 McCabe C, White PA, Hoole SP, et al. Right ventricular dysfunction in chronic thromboembolic obstruction of the pulmonary artery: a pressure-volume study using the conductance catheter. J Appl Physiol 2014; 116: 355-363.

11 Axell RG, Messer SJ, White PA, et al. Ventriculo-arterial coupling detects occult RV dysfunction in chronic thromboembolic pulmonary vascular disease. Physiol Rep 2017; 5: e13227. 